\title{
Diffusion tensor imaging study of pediatric patients with congenital hydrocephalus: 1-year postsurgical outcomes
}

\author{
Francesco T. Mangano, DO,,5 Mekibib Altaye, PhD, ${ }^{2,5}$ Robert C. McKinstry, MD, PhD, ${ }^{6,9}$ \\ Joshua S. Shimony, MD, PhD, , ${ }^{6,9}$ Stephanie K. Powell, PhD, 7,,10 Jannel M. Phillips, PhD, 4,5 \\ Holly Barnard, PhD, ${ }^{4,5}$ David D. Limbrick Jr., MD, PhD, ${ }^{8,9}$ Scott K. Holland, PhD, ${ }^{3,5}$ \\ Blaise V. Jones, MD, ${ }^{3,5}$ Jonathan Dodd, PsyD, ${ }^{7,9,10}$ Sarah Simpson, BS, ${ }^{3}$ Deanna Mercer, BS, ${ }^{8,9}$ \\ Akila Rajagopal, MS, ${ }^{3}$ Sarah Bidwell, MA, ${ }^{3}$ and Weihong Yuan, $\mathrm{PhD}^{3,5}$
}

1Division of Pediatric Neurosurgery, ${ }^{2}$ Division of Biostatistics and Epidemiology, ${ }^{3}$ Department of Radiology, and ${ }^{4}$ Division of Developmental and Behavioral Pediatrics-Psychology, Cincinnati Children's Hospital Medical Center, Cincinnati; ${ }^{5}$ University of Cincinnati College of Medicine, Cincinnati, Ohio; ${ }^{6}$ Mallinckrodt Institute of Radiology, ${ }^{7}$ Department of Neurology, and ${ }^{8}$ Department of Neurological Surgery, ${ }^{9}$ Washington University School of Medicine in St. Louis; and ${ }^{10}$ Department of Psychology, St. Louis Children's Hospital, St. Louis, Missouri

OBJECTIVE The purpose of this study was to investigate white matter (WM) structural abnormalities using diffusion tensor imaging (DTI) in children with hydrocephalus before CSF diversionary surgery (including ventriculoperitoneal shunt insertion and endoscopic third ventriculostomy) and during the course of recovery after surgery in association with neuropsychological and behavioral outcome.

METHODS This prospective study included 54 pediatric patients with congenital hydrocephalus ( 21 female, 33 male; age range 0.03-194.5 months) who underwent surgery and 64 normal controls (30 female, 34 male; age range 0.30197.75 months). DTI and neurodevelopmental outcome data were collected once in the control group and 3 times (preoperatively and at 3 and 12 months postoperatively) in the patients with hydrocephalus. DTI measures, including fractional anisotropy (FA), mean diffusivity (MD), axial diffusivity (AD), and radial diffusivity (RD) values were extracted from the genu of the corpus callosum (gCC) and the posterior limb of internal capsule (PLIC). Group analysis was performed first cross-sectionally to quantify DTI abnormalities at 3 time points by comparing the data obtained in the hydrocephalus group for each of the 3 time points to data obtained in the controls. Longitudinal comparisons were conducted pairwise between different time points in patients whose data were acquired at multiple time points. Neurodevelopmental data were collected and analyzed using the Adaptive Behavior Assessment System, Second Edition, and the Bayley Scales of Infant Development, Third Edition. Correlation analyses were performed between DTI and behavioral measures.

RESULTS Significant DTI abnormalities were found in the hydrocephalus patients in both the gCC (lower FA and higher $M D, A D$, and $R D$ ) and the PLIC (higher FA, lower AD and RD) before surgery. The DTI measures in the gCC remained mostly abnormal at 3 and 12 months after surgery. The DTI abnormalities in the PLIC were significant in FA and AD at 3 months after surgery but did not persist when tested at 12 months after surgery. Significant longitudinal DTI changes in the patients with hydrocephalus were found in the gCC when findings at 3 and 12 months after surgery were compared. In the PLIC, trend-level longitudinal changes were observed between preoperative findings and 3-month postoperative findings, as well as between 3- and 12-month postoperative findings. Significant correlation between DTI and developmental outcome was found at all 3 time points. Notably, a significant correlation was found between DTI in the PLIC at 3 months after surgery and developmental outcome at 12 months after surgery.

CONCLUSIONS The data showed significant WM abnormality based on DTI in both the gCC and the PLIC in patients with congenital hydrocephalus before surgery, and the abnormalities persisted in both the gCC and the PLIC at 3 months after surgery. The DTI values remained significantly abnormal in the gCC at 12 months after surgery. Longitudinal analy-

ABBREVIATIONS ABAS-II = Adaptive Behavior Assessment System, Second Edition; AD = axial diffusivity; Bayley III = Bayley Scale of Infant Development, Third Edition; CCHMC = Cincinnati Children's Hospital Medical Center; DTI = diffusion tensor imaging; FA = fractional anisotropy; FDR = false discovery rate; FOHR = frontal and occipital horn ratio; $\mathrm{GAC}=$ general adaptive composite; $\mathrm{gCC}=$ genu of corpus callosum; ICP = intracranial pressure; $\mathrm{MD}=$ mean diffusivity; PLIC = posterior limb of internal capsule; $\mathrm{RD}=$ radial diffusivity; $\mathrm{ROI}=$ region of interest; $\mathrm{SLCH} /$ WashU = St. Louis Children's Hospital and Washington University; WM = white matter.

SUBMITTED October 24, 2015. ACCEPTED February 11, 2016.

INCLUDE WHEN CITING Published online May 20, 2016; DOI: 10.3171/2016.2.PEDS15628. 
sis showed signs of recovery in both WM structures between different time points. Combined with the significant correlation found between DTI and neuropsychological measures, the findings of this study suggest that DTI can serve as a sensitive imaging biomarker for underlying neuroanatomical changes and postsurgical developmental outcome and even as a predictor for future outcomes.

http://thejns.org/doi/abs/10.3171/2016.2.PEDS15628

KEY WORDS hydrocephalus; DTI; white matter injury; postsurgery; outcome

$\mathrm{F}$ OR pediatric patients with hydrocephalus, CSF diversionary surgery (including ventriculoperitoneal shunt insertion and endoscopic third ventriculostomy) is the standard of care that can relieve clinical signs and symptoms of enlarged ventricles and increased intracranial pressure (ICP). Surgery has significantly reduced morbidity and mortality associated with hydrocephalus; ${ }^{25}$ however, despite advances in technology, the condition remains an incurable lifelong disorder. The rapid postoperative reversal of acute clinical symptoms does not correspond to a complete reversal of structural damage in the brain. Accumulating evidence has shown that there is no significant correlation between ventricle volume and neurocognitive outcome. ${ }^{11,30}$ The neuroanatomical changes that occurred prior to surgery may remain or continue to progress, leading to long-term behavioral and neuropsychological deficits. ${ }^{32}$ Data from both human and animal research have suggested that extensive areas of white matter (WM) fibers connecting various functionally important cortical and subcortical regions are vulnerable targets of injury as a result of ventricular enlargement and increased ICP in hydrocephalus. ${ }^{13,14,17,20,29,33,35}$

Diffusion tensor imaging (DTI) is an advanced MRI technique that uses diffusion properties of water molecules as probes to examine tissue structure, revealing characteristics of its microscopic organization. Anisotropic diffusion properties, as measured by DTI, are strongly influenced by the microstructural components of $\mathrm{WM}^{9}$ and provide a direct indication of the integrity of these components. Recent neuroimaging studies have shown that DTI can provide imaging biomarkers for hydrocephalus outcome. ${ }^{43}$ Cross-sectional studies have shown that DTI parameters can differentiate brain structures in patients with hydrocephalus from those of controls without hydrocephalus in both human and animal studies. ${ }^{15,22,26,30,34,38,40-42,44}$ Initial longitudinal studies have shown variable degrees of tendency for DTI parameters to return to a normal range after surgery. ${ }^{2,6,27,36}$ However, there is also strong evidence from both clinical and experimental studies suggesting that the damage to brain structures suffered before CSF diversion may be irreversible. . $^{5,12,13,16,19,29,31,32}$

Here we report the results of a prospective, longitudinal neuroimaging study combined with neuropsychological outcome assessment in pediatric patients with hydrocephalus. Data were acquired prior to CSF diversionary surgery and again at 3 and 12 months after surgery. The overall goal of this study was to establish the WM anisotropic diffusion property derived from DTI as a marker for in vivo WM damage and its course of recovery in children with hydrocephalus. These data allow us to have a better understanding of the variability in outcomes and provide a noninvasive and quantifiable means to predict these outcomes. The following hypotheses were tested and confirmed. 1) Pediatric patients with congenital hydrocephalus have abnormal anisotropic diffusion properties in WM prior to surgery. 2) The abnormal diffusion property in WM will recover with trends toward the normal range after surgery. 3) The WM anisotropic diffusion properties correlate significantly with outcome measures.

The findings of this study provide a necessary step toward establishing DTI as a significant clinical tool in the management of patients with hydrocephalus.

\section{Methods \\ Study Design and Participants}

All participants were enrolled in a federally funded, multicenter, prospective, longitudinal imaging study of pediatric hydrocephalus aimed at investigating neuroimaging and neurobehavioral outcomes at baseline prior to surgical management and at 3- and 12-month postoperative followup assessments. Between December 2009 and June 2014, a total of 146 infants, children, and adolescents, including 74 patients who were potential candidates for surgical treatment of hydrocephalus (age range 0.03-194.49 months) and 72 infants, children, and adolescents without hydrocephalus who served as controls (age range 0.56-197.75 months), were recruited from the 2 participating hospitals, Cincinnati Children's Hospital Medical Center (CCHMC) and St. Louis Children's Hospital and Washington University (SLCH/WashU). Participants' legal guardians gave written consent when the children were enrolled into the study. Participants older than 11 years also provided oral assent. The study was conducted according to institutional review board guidelines at both institutions.

For patients in the hydrocephalus group, the inclusion criteria were as follows: age 0-18 years; referral to radiology for brain MRI for evaluation of hydrocephalus and later referral for CSF diversion surgery; hydrocephalus with ventriculomegaly; and no evidence of other medical diagnoses that would predispose him/her to adverse neurological outcomes (e.g., preterm birth with severe intraventricular hemorrhage, Dandy-Walker syndrome, stroke, or spina bifida). Of the 74 patients with hydrocephalus, 20 were excluded from the study. Of these 20 patients, 11 were excluded because they did not receive surgical treatment for hydrocephalus, and 7 were excluded because they did not meet inclusion criteria. One family withdrew consent for their child's participation in the study. One additional child was excluded after being diagnosed with neurofibromatosis Type 1 . This resulted in a study population of 54 patients (21 female, 33 male; age range 0.03-194.49 months) with congenital hydrocephalus for the final data analysis. Detailed demographic information and etiology are included in Supplemental Table S1. 
The participants in the control group were recruited to match the patients in the hydrocephalus group in age and sex as closely as possible. They came from 2 sources: 1 ) pediatric patients who were referred for clinical MRI that was evaluated as normal by clinical neuroradiologists (the inclusion criteria for participants in the control group from this source were age $0-18$ years; referral to radiology for nonspecific symptoms not clearly related to a neurological disorder [e.g., headaches]; normal findings on MRI; no clinical or radiographic history of neurological or psychological disorders prior to the scan; no evidence of any neurological disorders [e.g., epilepsy, stroke]; or no suggestion of WM-related brain pathology within 3 months after the initial MRI, based on review of the patient's medical record) and 2) healthy individuals recruited from the general healthy population (the inclusion criteria for controls from this latter source were similar to those for the previously described subgroup). The MR images from these healthy controls were acquired under the research protocol on the same MRI scanners without sedation. Of the 72 control participants initially enrolled, 1 child was excluded after the diagnosis of epilepsy. Four children were excluded because of abnormal MRI findings. Two children were disqualified for having standard scores below 70 on either the Adaptive Behavior Assessment System, Second Edition (ABAS-II), ${ }^{21}$ or the Bayley Scales of Infant Development, Third Edition (Bayley-III). ${ }^{8}$ One additional child was excluded for poor image quality. This left a control population of 64 children (30 female, 34 male; age range 0.30-197.75 month) for further analysis.

Neuroimaging (MRI/DTI) and neuropsychological data were acquired in children with hydrocephalus at 3 time points: prior to the CSF diversionary surgery, 3 months after surgery, and 12 months after surgery. Neuroimaging data and neuropsychological data were acquired only once in children in the control group. Data were not acquired for some of the participants at some of the time points, most often due to the short window between diagnosis and surgery. In addition, depending on the severity of hydrocephalus, the ventricle enlargement was too severe in some patients to allow for reliable delineation of region of interest. The total numbers of participants for whom useful neuroimaging and neuropsychological outcome data were obtained are listed in Table 1.

\section{MRI Acquisition and Preprocessing}

MRI data were all acquired on 1.5-T scanners at either CCHMC (GE Signa, GE Healthcare) or SLCH/WashU (Siemens Avanto). A 15-direction diffusion-weighted spin-echo DTI sequence with single-shot echo planar imaging was used with the following specifications: FOV $240 \times 240 \mathrm{~mm}$, matrix $96 \times 96$, resolution $2.5 \times 2.5 \times 2.5$ $\mathrm{mm}$, number of slices 76, TR/TE 9400/93.2 msec, ASSET or IPAT factor 2, number of averages $2, \mathrm{~b}=1000 \mathrm{sec} / \mathrm{mm}^{2}$. One additional image with no diffusion weighting $(b=$ 0 ) was also acquired. The MR compatibility across the 2 sites (two 1.5-T GE Signa scanners from CCHMC and one 1.5-T Siemens Avanto from SLCH) was established using both an MR phantom (fBIRN phantom) and a traveling human phantom prior to the start of the participant enrollment. The compatibility was tested annually with the
TABLE 1. Number of data sets generated for different ROIs and outcome measures at different time points

\begin{tabular}{lcccc}
\hline & & \multicolumn{3}{c}{ Patients w/ HCP } \\
\cline { 3 - 5 } Outcome Measure & Controls & Preop & $\begin{array}{c}\text { P Mos } \\
\text { Postop }\end{array}$ & $\begin{array}{c}12 \text { Mos } \\
\text { Postop }\end{array}$ \\
\hline Neuroimaging: MRI/DTI & & & & \\
\hline gCC & 64 & 22 & 28 & 29 \\
\hline Left PLIC & 64 & 22 & 29 & 30 \\
\hline Neuropsych: ABAS-II & & & & \\
\hline GAC & 53 & 26 & 43 & 28 \\
\hline Conceptual & 53 & 26 & 43 & 28 \\
\hline Social & 54 & 27 & 44 & 28 \\
\hline Practical & 54 & 27 & 41 & 28 \\
\hline Motor Scale & 48 & 23 & 40 & 23 \\
\hline Neuropsych: Bayley-III & & & & \\
\hline Social & 33 & 7 & 27 & 17 \\
\hline Cognitive & 40 & 6 & 34 & 19 \\
\hline Language & 40 & 5 & 34 & 19 \\
\hline Motor & 39 & 4 & 34 & 19 \\
\hline
\end{tabular}

$\mathrm{HCP}=$ hydrocephalus.

same MR phantom and human phantom on all scanners used in the study. In addition, ACR (American College of Radiology) phantoms were also used for quality assurance within a week of each participant's scan to ensure the stability of scanner performance (Yuan et al., unpublished presentation, 2011)..$^{34,44}$

Image processing and analysis were all performed using the DTIStudio 3.02 software. ${ }^{28}$ Head motion and eddy current artifact were corrected using the automatic image registration method ${ }^{39}$ embedded in the DTIStudio software. ${ }^{28}$ An automatic bad slice detection method followed by additional visual inspection was used to identify signal dropout on diffusion-weighted images. DTI metrics including FA, MD, AD, and RD were calculated using the standard technique? Two regions of interest (ROIs) were examined in this study: the genu of the corpus callosum (gCC) and the posterior limb of the internal capsule (PLIC). The 2 ROIs were delineated based on the approach of Hermoye et al., ${ }^{23}$ as well as our previous work. ${ }^{2,40,44}$ It should be noted that, because shunt artifact was seen in postsurgery DTI images from many patients who were treated with adjustable valves (almost always on the right side), a bilateral DTI measurement in the PLIC was not always feasible at all 3 time points. Therefore, DTI data from the PLIC were extracted only from the left hemisphere in the present study.

\section{Ventricle Size Assessment}

Ventricle size was quantified using the frontal and occipital horn ratio (FOHR $)^{45}$ in the controls and in the participants with hydrocephalus at all 3 time points.

\section{Neuropsychological Outcome Measures}

The ABAS- $\mathrm{II}^{21}$ and the Bayley-III ${ }^{8}$ were included in the present study. Both tests were completed by pediatric neu- 
ropsychologists or by psychometrists supervised by pediatric neuropsychologists at either the Division of Developmental and Behavioral Pediatrics at CCHMC or the Department of Psychology at SLCH/WashU. The ABAS-II is a caregiver report form for adaptive skills comprising an overall adaptive score, the General Adaptive Composite (GAC), and 3 subscales assessing Conceptual, Social, and Practical skills. It also includes a subtest assessing motor skills. The Bayley-III is a direct assessment tool that measures early developmental skills; it assesses Cognitive, Language, and Motor skills for patients between 0.5 and 42 months of age. The Bayley-III also includes a caregiver report measure of early social-emotional behavior.

\section{Statistical Analysis}

Developmental changes in WM and DTI measures could significantly confound the results of the present study. Therefore, all the statistical analyses of DTI measures were corrected for age. We performed a regression analysis at each ROI for every DTI measure to remove the potential confounding effect of age. The residual values of the regression, defined as the difference between the actual DTI value from the individual participant and the simulated normal DTI value at the corresponding age based on the regression, were then used to substitute for the raw DTI values in the subsequent analysis. The group differences of DTI between patients and controls were first tested cross-sectionally for data obtained before surgery, 3 months after surgery, and 12 months after surgery separately using an independent 2-tailed unpaired t-test. The false discovery rate (FDR) method was applied at each time point to correct for potential false-positive findings resulting from multiple comparisons of DTI measures and ROIs. Longitudinal comparisons of DTI abnormalities were tested for each pair of the 3 time points using a paired t-test in children with hydrocephalus who had longitudinal data at the corresponding time points. Pearson correlation analysis was performed to test the association between DTI measures and neuropsychological measures assessed at the same time and between DTI measures assessed at 3 months after surgery and neuropsychological measures assessed at 12 months after surgery. Due to the limitation in sample size in the longitudinal comparisons and correlation analyses, statistical significance was reported without correction for multiple comparisons in these analyses.

\section{Results \\ Ventricle Size and Correlation With DTI and Neuropsychological Measures}

The FOHR values in the control group ranged from 0.197 to 0.375 (mean $[ \pm \mathrm{SD}] 0.310 \pm 0.036$ ). Compared with the controls, patients with hydrocephalus had significantly larger ventricles, as expressed by FOHR values, before surgery (range $0.331-0.777$, mean $0.555 \pm 0.102$, $p$ $<0.0001$ ) and at both 3 (range 0.339-0.687, mean 0.483 $\pm 0.090, \mathrm{p}<0.0001$ ) and 12 months after surgery (range $0.265-0.579$, mean $0.400 \pm 0.089$, $\mathrm{p}<0.0001$ ). No significant correlation was found between FOHR and DTI measures or FOHR and neuropsychological measures in any group or at any time point.

\section{DTI Abnormalities in Children With Hydrocephalus}

The ROI-based DTI measures in patients with hydrocephalus at the preoperative and 3- and 12-month postoperative time points are displayed in Fig. 1 (for gCC) and Fig. 2 (for PLIC). As expected, there was a developmental change in the DTI values with age in both ROIs in the control group. The regression coefficients based on exponential model fit are included in Table 2.

Quantitatively, at both the preoperative time point and the 3-month postoperative time point, DTI parameters in the gCC in patients with hydrocephalus were found to be significantly different from those of controls, characterized by lower FA ( $p=0.0008$ for both time points), higher MD ( $\mathrm{p}=0.0004$ for both time points), higher AD $(\mathrm{p}=0.0095$ for the preoperative time point, $\mathrm{p}=0.0003$ for 3-month postoperative time point), and higher RD ( $\mathrm{p}$ $=0.0003$ for the preoperative time point, $\mathrm{p}=0.0002$ for 3 -month postoperative time point). Analysis of DTI parameters in the left PLIC showed that, in comparison with controls, the patients with hydrocephalus had significantly higher FA ( $\mathrm{p}=0.0002$ for the preoperative time point, $\mathrm{p}=0.0005$ for the 3 -month postoperative time point), higher AD ( $\mathrm{p}=0.0002$ for the preoperative time point, $\mathrm{p}=0.0003$ for the 3-month postoperative time point), and lower RD at the preoperative time point $(\mathrm{p}=0.0353)$. With respect to the 12-month postoperative time point, the analysis of DTI parameters in the gCC showed significantly lower FA $(p=0.0008)$, higher MD $(p=0.0004)$, and higher RD ( $\mathrm{p}=0.0003)$ in patients with hydrocephalus than in controls. No significance in AD was found between patients with hydrocephalus and the controls at 12 months after surgery. In the left PLIC, no significant between-group difference was found in any of the 4 DTI measures for the 12-month postoperative time point (Figs. 3 and 4; Table 3).

\section{Longitudinal Difference in DTI Abnormalities}

To perform longitudinal comparisons, we first quantified the DTI abnormalities as the difference between the actual DTI values in children with hydrocephalus and the normal DTI value at the corresponding age based on the regression of DTI data using the control data sets. Longitudinal DTI comparisons between different time points were made for those children with hydrocephalus who had multiple MRI/DTI data sets. As shown in Table 4, no significant longitudinal change in DTI abnormality (difference between patient DTI and simulated normal DTI at the same age) was found between the preoperative and 3 -month postoperative data for any of the 4 DTI parameters in the $\mathrm{gCC}(\mathrm{n}=10)$. Comparison of 3 - and 12 -month postoperative findings showed statistically significant decreases in DTI abnormalities with respect to FA ( $\mathrm{p}=$ $0.0417), \mathrm{MD}(\mathrm{p}=0.0049)$, and $\mathrm{RD}(\mathrm{p}=0.0025)$ in the $\mathrm{gCC}$ $(n=19)$. It should be noted that a decrease of DTI abnormalities means that the DTI values from patients with hydrocephalus become closer to the normal simulated value as shown in Fig. 5. Comparison of the preoperative and the 12-month postoperative assessment findings showed similar changes in the gCC $(\mathrm{n}=11)$, with statistical significance in MD ( $\mathrm{p}=0.0095)$ and $\mathrm{RD}(\mathrm{p}=0.0002)$ and a trend toward significance in FA $(p=0.078)$. In the left 

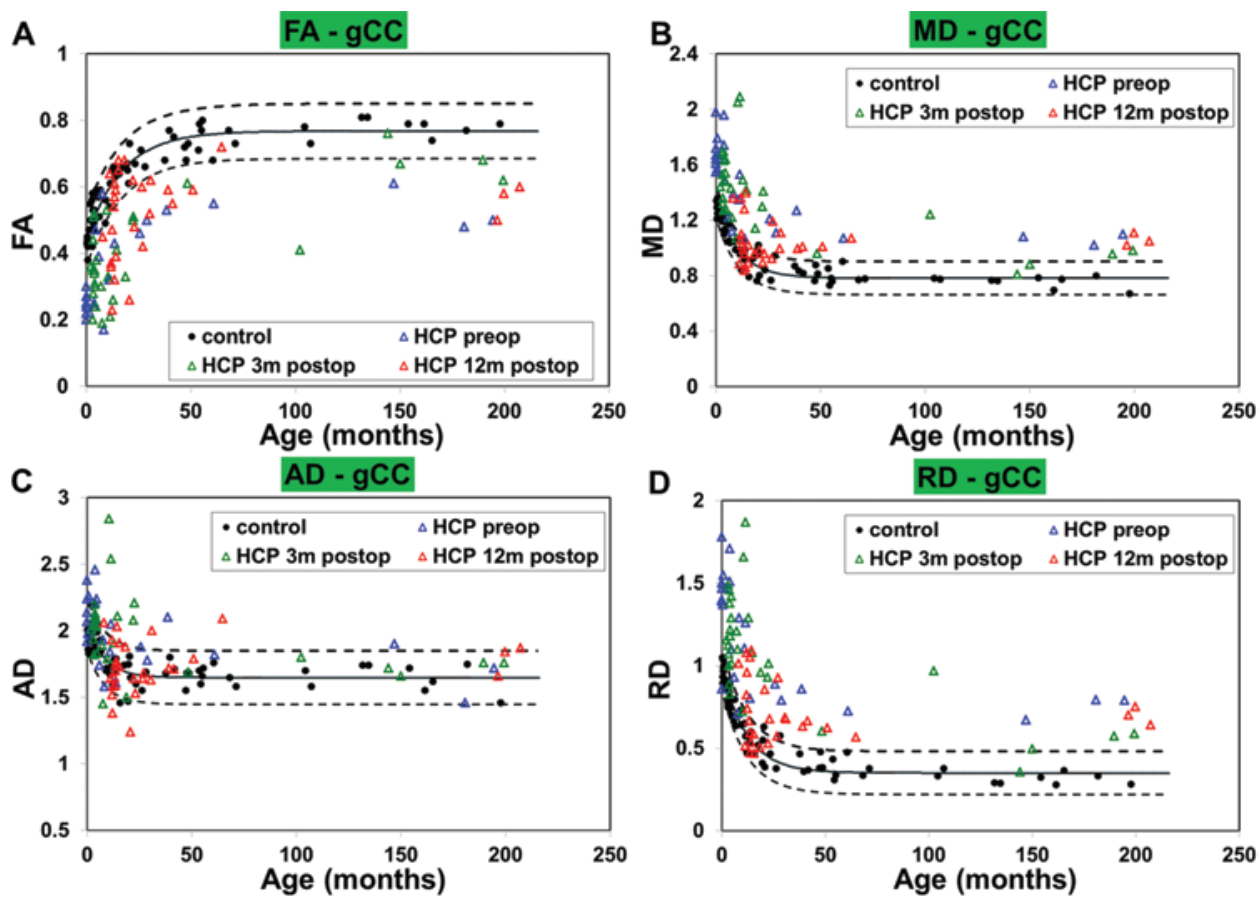

FIG. 1. Scatter plots showing the group differences in FA (A), MD (B), $A D(C)$, and $R D(D)$ in the gCC. Each symbol represents the DTI value extracted from the gCC from a single participant. The solid black circles, blue triangles, green triangles, and red triangles correspond to data obtained from participants in the control group, data obtained from patients before surgery, data obtained from patients 3 months after surgery, and data obtained from patients 12 months after surgery, respectively. The solid black line and the 2 dashed lines represent the results of regression analysis (the fitted curve and the two 95\% prediction intervals) based on the data from the control group. $m=$ months.
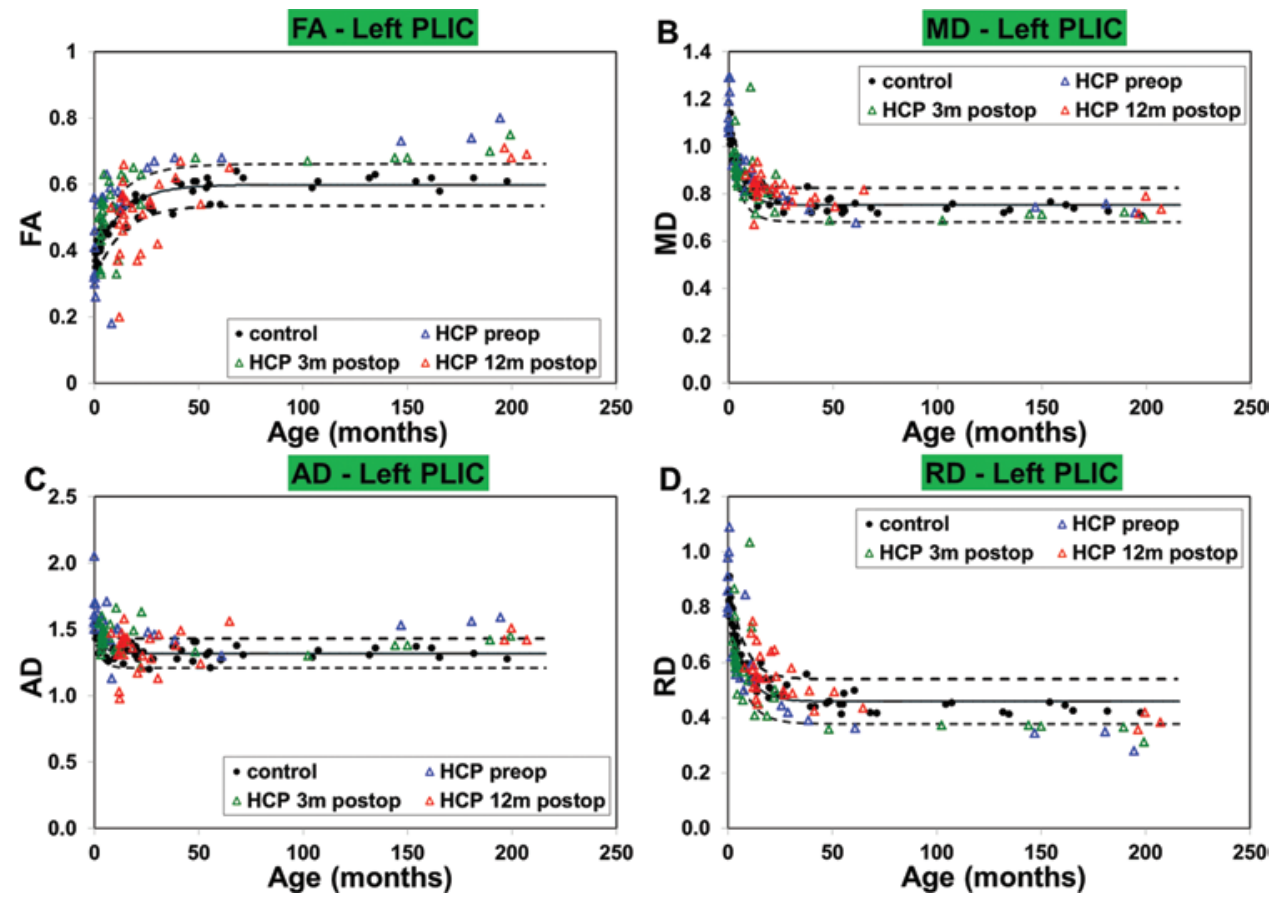

FIG. 2. Scatter plots showing the group difference of $F A(A), M D(B), A D(C)$, and $R D(D)$ in the left PLIC. Each symbol represents the DTI value extracted from the left PLIC from a single participant. The solid black circles, blue triangles, green triangles, and red triangles correspond to data obtained from participants in the control group, data obtained from patients before surgery, data obtained from patients 3 months after surgery, and data obtained from patients 12 months after surgery, respectively. The solid black line and the two dashed lines represent the results of regression analysis (the fitted curve and the two $95 \%$ prediction intervals) based on the data from the control group. 
TABLE 2. Results of regression analysis between normal DTI measures and age using an exponential model*

\begin{tabular}{|c|c|c|c|c|c|}
\hline \multirow[b]{2}{*}{ Parameter } & \multicolumn{4}{|c|}{ Regression Coefficients } & \multirow[b]{2}{*}{$R^{2} \dagger$} \\
\hline & a & $b$ & c & $y$-Intercept & \\
\hline \multicolumn{6}{|l|}{$\mathrm{gCC}$} \\
\hline FA & -0.3266 & 0.0588 & 0.7677 & 0.4411 & 0.9029 \\
\hline MD & 0.5544 & 0.0937 & 0.7820 & 1.3364 & 0.9246 \\
\hline$A D$ & 0.4065 & 0.1215 & 1.6482 & 2.0545 & 0.6828 \\
\hline $\mathrm{RD}$ & 0.6315 & 0.0829 & 0.3506 & 0.9821 & 0.9307 \\
\hline \multicolumn{6}{|l|}{ Left PLIC } \\
\hline FA & -0.2236 & 0.0748 & 0.5989 & 0.3753 & 0.8804 \\
\hline MD & 0.3335 & 0.1816 & 0.7522 & 1.0875 & 0.9088 \\
\hline$A D$ & 0.2428 & 0.3218 & 1.3191 & 1.5618 & 0.6528 \\
\hline $\mathrm{RD}$ & 0.3801 & 0.1414 & 0.4592 & 0.8391 & 0.9174 \\
\hline
\end{tabular}

* The regression model $\left(Y=a^{*} \exp \left[-b^{*} X+c\right]\right)$ fit the data in all ROls with $a$ significance level of $p<0.001$.

$\dagger$ Exponential model fit.

PLIC, as in the gCC, no statistically significant change was found in DTI abnormality in any of the 4 DTI parameters when preoperative and 3-month postoperative values were compared. Comparison of 3- and 12-month postoperative findings showed a trend-level change in DTI abnormality for FA ( $p=0.0590)$ but not for the other 3 parameters. Comparison of preoperative and 12-month postoperative findings (Fig. 6; Table 4) showed a trend-level change in DTI abnormalities for FA $(p=0.0594)$ AD $(p=0.0650)$, and RD ( $\mathrm{p}=0.0717)$.

\section{Abnormalities in Neuropsychological Measures}

The results from the present sample are summarized in Table 5. Briefly, in comparison with the controls, patients with hydrocephalus had scores that were significantly lower (or at least showed a trend toward being significantly lower) in GAC, all 3 subscale scores, and motor skills at all 3 time points, with the only exception being the Practical subscale score from the 3-month postoperative time point based on the caregiver assessment in ABAS-II tests. In the Bayley-III test, patients with hydrocephalus showed a trend toward lower scores in Social Emotional skill at both postoperative time points and had significantly lower scores in Cognitive, Language, and Motor skills at all 3 time points.

\section{Correlation of DTI With Neuropsychological Measures at the Time of Imaging}

In the control group, no significant correlation was found between any DTI measure in the gCC or the left PLIC and any neuropsychological measure in either ABAS-II or Bayley-III. Analysis of preoperative imaging in patients with hydrocephalus showed a statistically significant positive correlation between the FA residual in the $\mathrm{gCC}$ and the Motor Scale score in ABAS-II $(\mathrm{r}=0.79, \mathrm{n}=$ $10, p=0.007$; Fig. 7A). Analysis of imaging data obtained 3 months after surgery in patients with hydrocephalus showed a statistically significant positive correlation between FA residual in the gCC and GAC ( $\mathrm{r}=0.43, \mathrm{n}=26, \mathrm{p}$ $=0.028$; Fig. 7B), Motor Scale score $(r=0.45, n=22, p=$ $0.034)$ in ABAS-II, and Language score $(\mathrm{r}=0.61, \mathrm{n}=19, \mathrm{p}$ $=0.005$; Fig. 7C) in Bayley-III; RD in gCC was inversely correlated with Motor Scale score $(\mathrm{r}=-0.44, \mathrm{n}=22, \mathrm{p}$
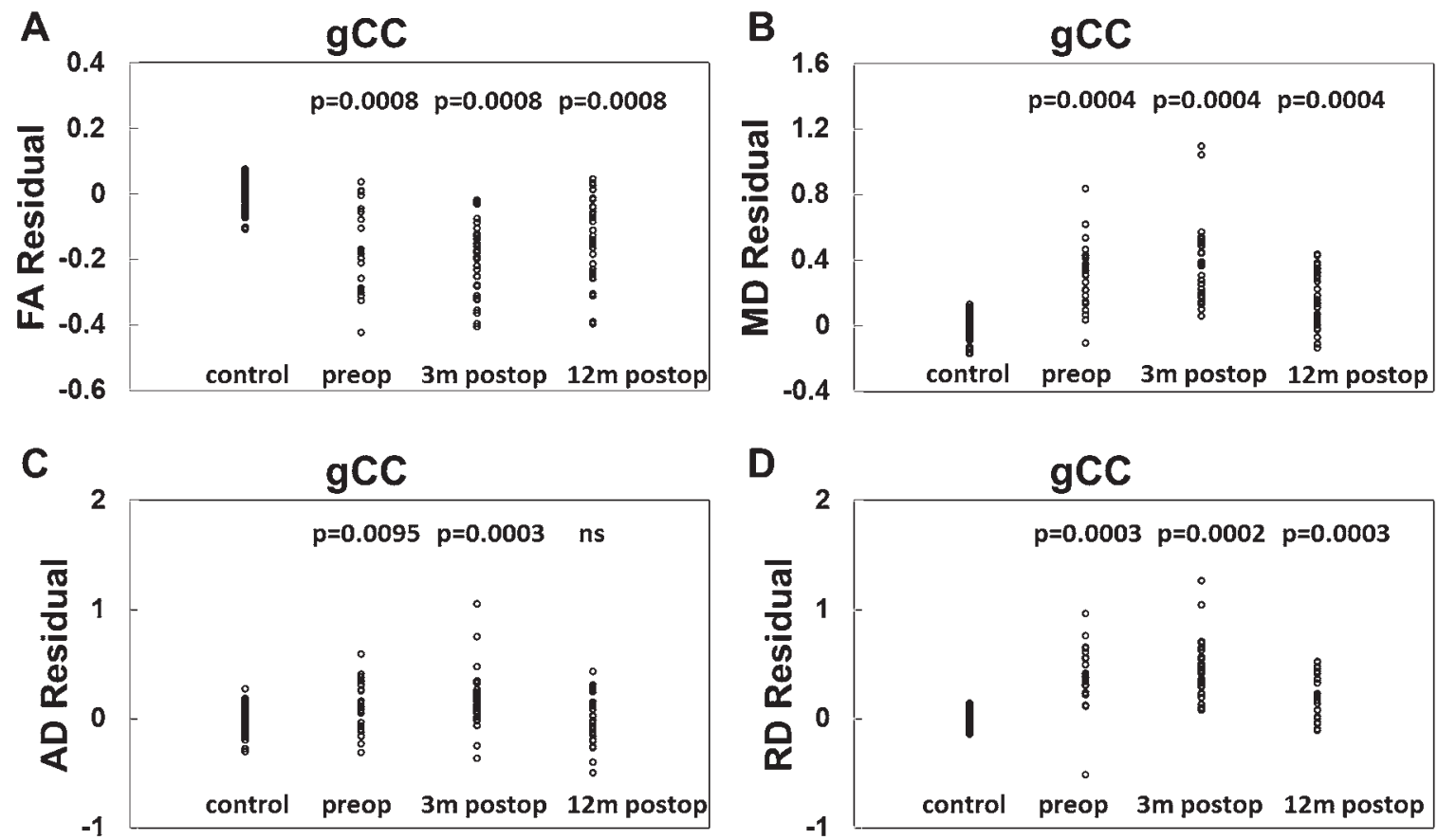

FIG. 3. Cross-sectional comparisons of DTI residual in the gCC between imaging studies obtained in the controls and studies obtained in hydrocephalus patients at 3 different time points. A: FA. B: MD. C: AD. D: RD. ns = not significant. 

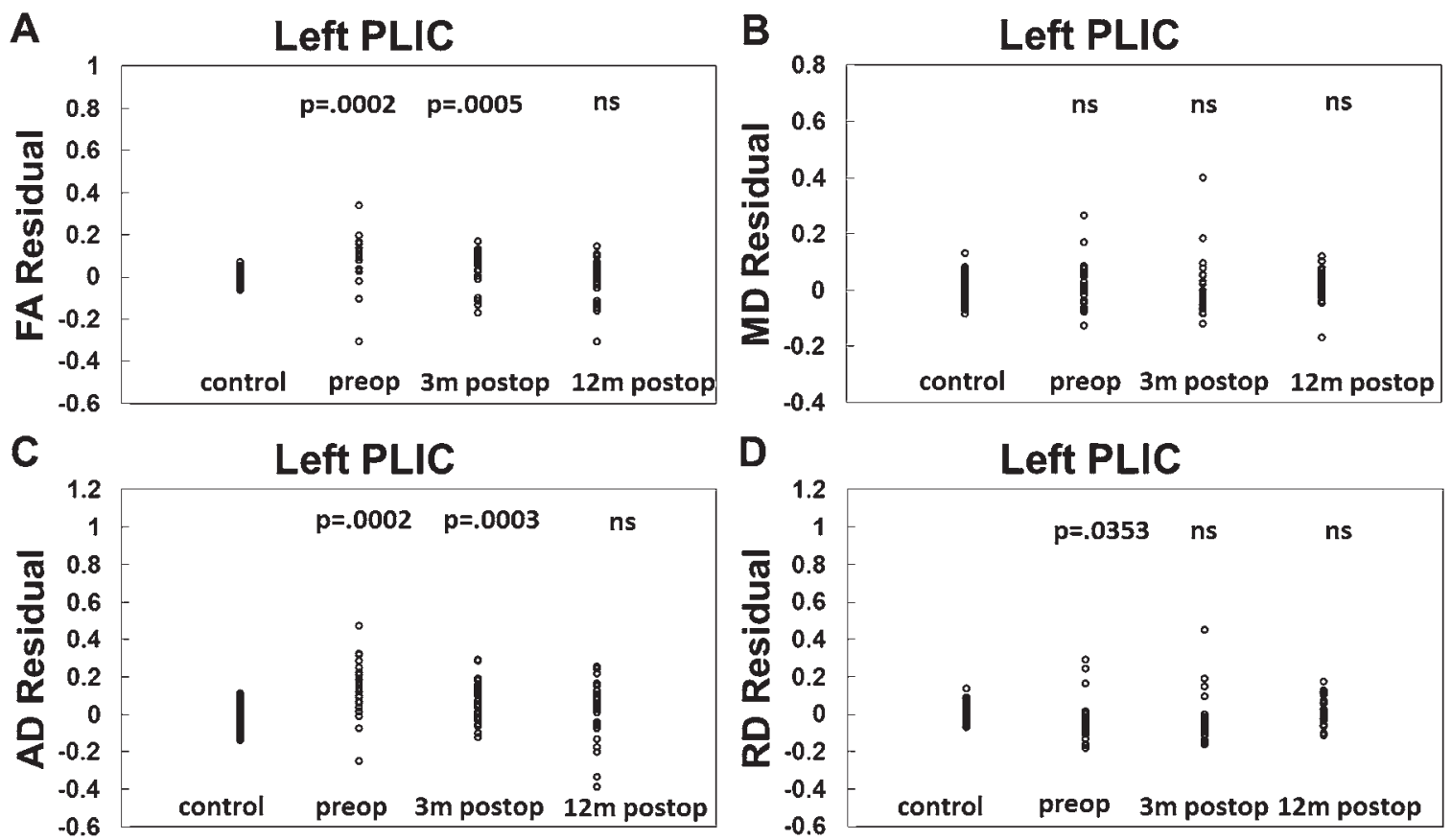

FIG. 4. Cross-sectional comparisons of DTI residual in the left PLIC between controls and hydrocephalus patients at 3 different time points. A: FA. B: MD. C: AD. D: RD. ns = not significant.

$=0.041)$ in ABAS-II. Analysis of imaging data obtained 12 months after surgery in children with hydrocephalus showed a significant positive correlation between FA residual in the gCC and Motor Scale score in ABAS-II $(\mathrm{r}=$ $0.57, \mathrm{n}=17, \mathrm{p}=0.018)$, as well as Motor scores $(\mathrm{r}=0.72, \mathrm{n}$ $=14, \mathrm{p}=0.004$; Fig. 7D) in Bayley-III.

\section{Correlation of DTI Findings With Future Neuropsychological Outcome}

The FA value in the gCC measured 3 months after surgery in children with hydrocephalus was found to have a significant positive correlation with the ABAS-II GAC ( $\mathrm{r}$ $=0.638, n=15, p=0.011$; Fig. 8A), Conceptual score $(r=$ $0.633, \mathrm{n}=15, \mathrm{p}=0.011)$, Practical score $(\mathrm{r}=0.633, \mathrm{n}=15$, $\mathrm{p}=0.011)$, and Motor Scale score $(\mathrm{r}=0.716, \mathrm{n}=12, \mathrm{p}=$ 0.009; Fig. 8B) and Bayley-III Motor score ( $\mathrm{r}=0.842, \mathrm{n}=$ $10, p=0.002$; Fig. $8 \mathrm{C}$ ) obtained at 12 months after surgery. The RD in gCC measured 3 months after surgery was inversely correlated with the ABAS-II Motor Scale score (r $=-0.605, \mathrm{p}=0.037, \mathrm{n}=12)$ and Bayley-III Motor score $(\mathrm{r}$ $=-0.678, \mathrm{n}=10, \mathrm{p}=0.031$; Fig. 8D) obtained 12 months after surgery.

\section{Discussion}

The use of DTI for the noninvasive evaluation of clinical hydrocephalus was first reported by Assaf et al. in $2006 .{ }^{6}$ Since that time, the evaluation of WM tracts to better understand pediatric hydrocephalus has been applied both prospectively and retrospectively in human and animal studies. Significant WM abnormalities in the corpus callosum and internal capsule, i.e., decreased FA in the corpus callosum and increased FA in the internal capsule (interpreted as disruption and compression, respectively) have been reported by our group, as well as others., $3,6,22,40,44$ Recovery after CSF diversionary surgery, with a trend toward normalization, has also been reported. ${ }^{2,6}$ It is interesting and worth noting that a different trend was identified when patients with benign external hydrocephalus were analyzed. Those patients also had various DTI abnormalities in initial screening MRI studies. In the patients with follow-up imaging, however, no WM abnormalities were detected, and there was no need of surgical intervention. ${ }^{37}$ More recently, we reported preliminary findings suggesting a correlation between abnormal DTI values and motor deficits in a prospectively studied patient population (a subset of the patients in the present study, studied at the preoperative stage ${ }^{44}$ ). Other groups have also reported that DTI parameters are sensitive in the study of hydrocephalus and suggest that DTI could serve as an important tool for quantitative assessment. Others have noted that, in comparison with ventricular size, DTI may offer a better association of WM injury and neurological deficits compared with ventricular size. ${ }^{10,30}$

In the present study, DTI and neurophysiological outcomes were investigated in pediatric patients with surgically treated congenital hydrocephalus in a prospective manner, first before surgery and then at 3 and 12 months after surgery. The data collected in the present study supported the hypothesis that preoperative DTI measures were abnormal in patients with hydrocephalus in comparison with the findings in age-matched controls. These data are consistent with our previous reports, as well as those of other groups..$^{2,10,20,44}$ In general, FA values are low in the $\mathrm{gCC}$ and high in the PLIC. 
TABLE 3. Cross-sectional comparisons of DTI residual between controls and patients with hydrocephalus at 3 different time points

\begin{tabular}{|c|c|c|c|c|c|c|c|}
\hline \multirow{2}{*}{$\begin{array}{l}\text { Time Point \& } \\
\text { Parameter }\end{array}$} & \multicolumn{2}{|c|}{ Mean \pm SD } & \multicolumn{2}{|c|}{ Difference Btwn Groups } & \multirow[b]{2}{*}{$\mathrm{df}$} & \multirow[b]{2}{*}{$t$} & \multirow[b]{2}{*}{ p Value* } \\
\hline & Controls & Patients w/ HCP & Mean & $95 \% \mathrm{Cl}$ & & & \\
\hline \multicolumn{8}{|l|}{ Preop } \\
\hline \multicolumn{8}{|l|}{ gCC } \\
\hline FA & $-0.0005 \pm 0.0414$ & $-0.1743 \pm 0.1296$ & 0.1738 & 0.1374 to 0.2102 & 84 & 9.4956 & 0.0008 \\
\hline MD & $0.0006 \pm 0.0709$ & $0.3187 \pm 0.2065$ & -0.3181 & -0.3771 to -0.2590 & 84 & 10.7109 & 0.0004 \\
\hline$A D$ & $0.0000 \pm 0.1111$ & $0.1008 \pm 0.2203$ & -0.1008 & -0.1734 to 0.0281 & 84 & 2.7587 & 0.0095 \\
\hline $\mathrm{RD}$ & $0.0008 \pm 0.0687$ & $0.4004 \pm 0.2904$ & -0.3996 & -0.4767 to -0.3225 & 84 & 10.3054 & 0.0003 \\
\hline \multicolumn{8}{|l|}{ Left PLIC } \\
\hline FA & $-0.0001 \pm 0.0309$ & $0.0828 \pm 0.1210$ & -0.0829 & -0.1154 to -0.0504 & 84 & 5.0694 & 0.0002 \\
\hline MD & $0.0011 \pm 0.0431$ & $0.0227 \pm 0.0852$ & -0.0228 & -0.0475 to 0.0019 & 84 & 1.8368 & NS \\
\hline$A D$ & $-0.0000 \pm 0.0624$ & $0.1469 \pm 0.1476$ & -0.1470 & -0.1919 to -0.1020 & 84 & 6.5021 & 0.0002 \\
\hline $\mathrm{RD}$ & $0.0004 \pm 0.0436$ & $-0.0388 \pm 0.1232$ & 0.0392 & -0.0037 to 0.0747 & 84 & 2.1948 & 0.0353 \\
\hline \multicolumn{8}{|l|}{3 mos postop } \\
\hline \multicolumn{8}{|l|}{ gCC } \\
\hline FA & $-0.0005 \pm 0.0414$ & $-0.1948 \pm 0.1041$ & 0.1942 & 0.1642 to 0.2243 & 90 & 12.8527 & 0.0008 \\
\hline MD & $0.0006 \pm 0.0709$ & $0.3609 \pm 0.2486$ & -0.3602 & -0.4271 to -0.2934 & 90 & 10.7034 & 0.0004 \\
\hline$A D$ & $0.0000 \pm 0.1111$ & $0.1850 \pm 0.2689$ & -0.1850 & -0.2634 to -0.1066 & 90 & 4.6881 & 0.0003 \\
\hline $\mathrm{RD}$ & $0.0008 \pm 0.0687$ & $0.4386 \pm 0.2734$ & -0.4380 & -0.5102 to -0.3658 & 90 & 12.0531 & 0.0002 \\
\hline \multicolumn{8}{|l|}{ Left PLIC } \\
\hline FA & $-0.0001 \pm 0.0309$ & $0.0430 \pm 0.0815$ & -0.0431 & -0.0662 to -0.0199 & 91 & 3.7001 & 0.0005 \\
\hline MD & $0.0011 \pm 0.0431$ & $0.0107 \pm 0.0949$ & -0.0109 & -0.0368 to 0.0152 & 91 & 0.8242 & NS \\
\hline$A D$ & $-0.0000 \pm 0.0624$ & $0.0663 \pm 0.1007$ & -0.0663 & -0.1002 to -0.0324 & 91 & 3.8833 & 0.0003 \\
\hline $\mathrm{RD}$ & $0.0004 \pm 0.0436$ & $-0.0264 \pm 0.1190$ & 0.0267 & -0.0068 to 0.0060 & 91 & 1.5850 & NS \\
\hline \multicolumn{8}{|l|}{12 mos postop } \\
\hline \multicolumn{8}{|l|}{ gCC } \\
\hline FA & $-0.0005 \pm 0.0414$ & $-0.1437 \pm 0.1251$ & 0.1432 & 0.1088 to 0.1777 & 91 & 8.2571 & 0.0008 \\
\hline MD & $0.0006 \pm 0.0709$ & $0.1337 \pm 0.1716$ & -0.1331 & -0.1829 to -0.0083 & 91 & 5.3101 & 0.0004 \\
\hline$A D$ & $0.0000 \pm 0.1111$ & $-0.0644 \pm 0.3955$ & 0.0628 & -0.0428 to 0.1684 & 91 & 1.1809 & NS \\
\hline $\mathrm{RD}$ & $0.0008 \pm 0.0687$ & $0.2031 \pm 0.2007$ & -0.2023 & -0.2579 to -0.1467 & 91 & 7.2219 & 0.0003 \\
\hline \multicolumn{8}{|l|}{ Left PLIC } \\
\hline FA & $-0.0001 \pm 0.0309$ & $0.0089 \pm 0.0958$ & 0.0088 & -0.0173 to 0.0350 & 92 & 0.6698 & NS \\
\hline MD & $0.0011 \pm 0.0431$ & $0.0113 \pm 0.0530$ & -0.0114 & -0.0287 to 0.0058 & 92 & 1.3147 & NS \\
\hline$A D$ & $-0.0000 \pm 0.0624$ & $0.0137 \pm 0.1531$ & -0.0138 & -0.0578 to 0.0303 & 92 & 0.6198 & NS \\
\hline $\mathrm{RD}$ & $0.0004 \pm 0.0436$ & $0.0090 \pm 0.0683$ & -0.0087 & -0.0318 to 0.0145 & 92 & 0.7444 & NS \\
\hline
\end{tabular}

$\mathrm{df}=$ degrees of freedom; $\mathrm{NS}=$ not significant.

* $p$ values corrected using the FDR method.

\section{Persistence of DTI Abnormalities During the 1st Year After Surgery}

It was hypothesized that there would be significant trends toward recovery in terms of DTI measures in the WM at follow-up examinations after the initial surgical procedure. However, this hypothesis was not proved to be entirely true. Qualitatively, abnormal DTI values seem to persist in many patients (Figs. 1 and 2). Cross-sectionally, the DTI findings in the corpus callosum of hydrocephalus patients demonstrated persistence of abnormalities both at 3- and 12-month follow-up compared with findings in controls. In contrast, the diffusion abnormalities in the PLIC were significant in studies performed 3 months after surgery, but the abnormalities were not appreciated at 12 months after surgery (Figs. 3 and 4). Longitudinally, the gCC DTI abnormalities in patients with hydrocephalus (difference between the hydrocephalus patients' data and the controls' data for the gCC) did not differ significantly between the preoperative and 3-month postoperative imaging studies, suggesting persistence of WM injury during the first 3 months after surgery despite the decreased ventricle size and improvement in clinical symptoms. However, a significant longitudinal difference was observed with respect to all 4 DTI measures in the gCC between the 3and 12-month postoperative studies, with DTI values closer to normal in the 12-month postoperative assessments. Significant or trend-level differences in DTI abnormalities in the gCC between preoperative and 12-month postop- 
TABLE 4. Longitudinal comparisons of DTI residual at 3 different time points in patients with hydrocephalus

\begin{tabular}{|c|c|c|c|c|c|c|c|c|}
\hline Measurement & $\mathrm{N}$ & Mean & SD & SEM & Min & Median & Max & $p$ Value \\
\hline \multicolumn{9}{|l|}{ gCC } \\
\hline \multicolumn{9}{|c|}{3 mos postop - preop } \\
\hline FA & 11 & -0.013 & 0.137 & 0.041 & -0.237 & 0.016 & 0.197 & NS \\
\hline MD & 11 & 0.064 & 0.324 & 0.098 & -0.458 & 0.010 & 0.737 & NS \\
\hline$A D$ & 11 & 0.135 & 0.373 & 0.113 & -0.522 & 0.110 & 0.985 & NS \\
\hline $\mathrm{RD}$ & 11 & 0.015 & 0.313 & 0.094 & -0.416 & -0.041 & 0.623 & NS \\
\hline \multicolumn{9}{|c|}{12 mos postop - 3 mos postop } \\
\hline FA & 18 & 0.050 & 0.096 & 0.023 & -0.104 & 0.043 & 0.224 & 0.0418 \\
\hline MD & 18 & -0.228 & 0.300 & 0.071 & -1.040 & -0.247 & 0.160 & 0.0049 \\
\hline$A D$ & 18 & -0.273 & 0.565 & 0.133 & -2.035 & -0.137 & 0.270 & 0.0565 \\
\hline $\mathrm{RD}$ & 18 & -0.237 & 0.284 & 0.067 & -0.935 & -0.224 & 0.186 & 0.0025 \\
\hline \multicolumn{9}{|c|}{12 mos postop - preop } \\
\hline FA & 10 & 0.031 & 0.046 & 0.015 & -0.054 & 0.025 & 0.101 & 0.0775 \\
\hline MD & 10 & -0.131 & 0.126 & 0.040 & -0.303 & -0.160 & 0.101 & 0.0095 \\
\hline$A D$ & 10 & -0.197 & 0.583 & 0.184 & -1.740 & -0.086 & 0.388 & NS \\
\hline $\mathrm{RD}$ & 10 & -0.177 & 0.092 & 0.029 & -0.312 & -0.199 & -0.029 & 0.0002 \\
\hline \multicolumn{9}{|l|}{ Left PLIC } \\
\hline \multicolumn{9}{|c|}{3 mos postop - preop } \\
\hline FA & 11 & -0.038 & 0.097 & 0.029 & -0.169 & -0.051 & 0.174 & NS \\
\hline MD & 11 & -0.023 & 0.057 & 0.017 & -0.145 & -0.030 & 0.064 & NS \\
\hline$A D$ & 11 & -0.098 & 0.144 & 0.043 & -0.364 & -0.119 & 0.231 & 0.0470 \\
\hline $\mathrm{RD}$ & 11 & 0.005 & 0.067 & 0.020 & -0.093 & 0.021 & 0.098 & NS \\
\hline \multicolumn{9}{|c|}{12 mos postop - 3 mos postop } \\
\hline FA & 19 & -0.040 & 0.083 & 0.019 & -0.223 & -0.028 & 0.117 & 0.0525 \\
\hline MD & 19 & -0.004 & 0.098 & 0.022 & -0.353 & 0.023 & 0.094 & NS \\
\hline$A D$ & 19 & -0.047 & 0.159 & 0.036 & -0.512 & -0.029 & 0.219 & NS \\
\hline $\mathrm{RD}$ & 19 & 0.029 & 0.100 & 0.023 & -0.251 & 0.030 & 0.207 & NS \\
\hline \multicolumn{9}{|c|}{12 mos postop - preop } \\
\hline FA & 11 & -0.096 & 0.150 & 0.045 & -0.365 & -0.065 & 0.146 & 0.0594 \\
\hline MD & 11 & 0.013 & 0.063 & 0.019 & -0.093 & 0.031 & 0.132 & NS \\
\hline$A D$ & 11 & -0.104 & 0.166 & 0.050 & -0.520 & -0.067 & 0.072 & 0.0650 \\
\hline $\mathrm{RD}$ & 11 & 0.073 & 0.119 & 0.036 & -0.137 & 0.084 & 0.304 & 0.0717 \\
\hline
\end{tabular}

erative imaging studies were also found, with DTI values close to the normal range at 12 months after surgery. In contrast, the DTI abnormality in the left PLIC in the patients with hydrocephalus (difference between the hydrocephalus patients' data and the controls' data for the left PLIC) appeared to resolve sooner after the surgery based on the longitudinal comparisons.

\section{Correlation Between DTI and Neuropsychological Measures and Predictive Value of DTI for Future Outcome Measures}

In the present study, we reported correlations between DTI measures and neuropsychological assessments in children with hydrocephalus. At all 3 time points, correlations were found between ABAS-II or Bayley-III in patients with hydrocephalus and their DTI results in the gCC (in general, positively with FA, negatively with RD). The direction of correlation is in line with our hypotheses-i.e., with a structure with abnormally lower FA and/or higher $\mathrm{MD}$ (or AD or RD), a patient with hydrocephalus performs better in the neuropsychological assessment when his or her DTI measure in the gCC is closer to the normal range. Although there is no direct evidence based on underlying neuroanatomy, the correlation indicates that, in addition to the persistent abnormality in DTI at both 3 and 12 months after surgery, the association between neuroanatomy and neuropsychological measures has not resolved within the time frame of this study (1 year after surgery). In addition to the correlation between outcome measures and contemporaneous DTI measures, significant correlations were also found between DTI findings obtained at 3 months after surgery and outcome measures 12 months after surgery, suggesting that behavioral and neuropsychological changes may be influenced by earlier WM changes. The predictive value of WM integrity based on DTI for future outcome has been reported frequently in populations such 

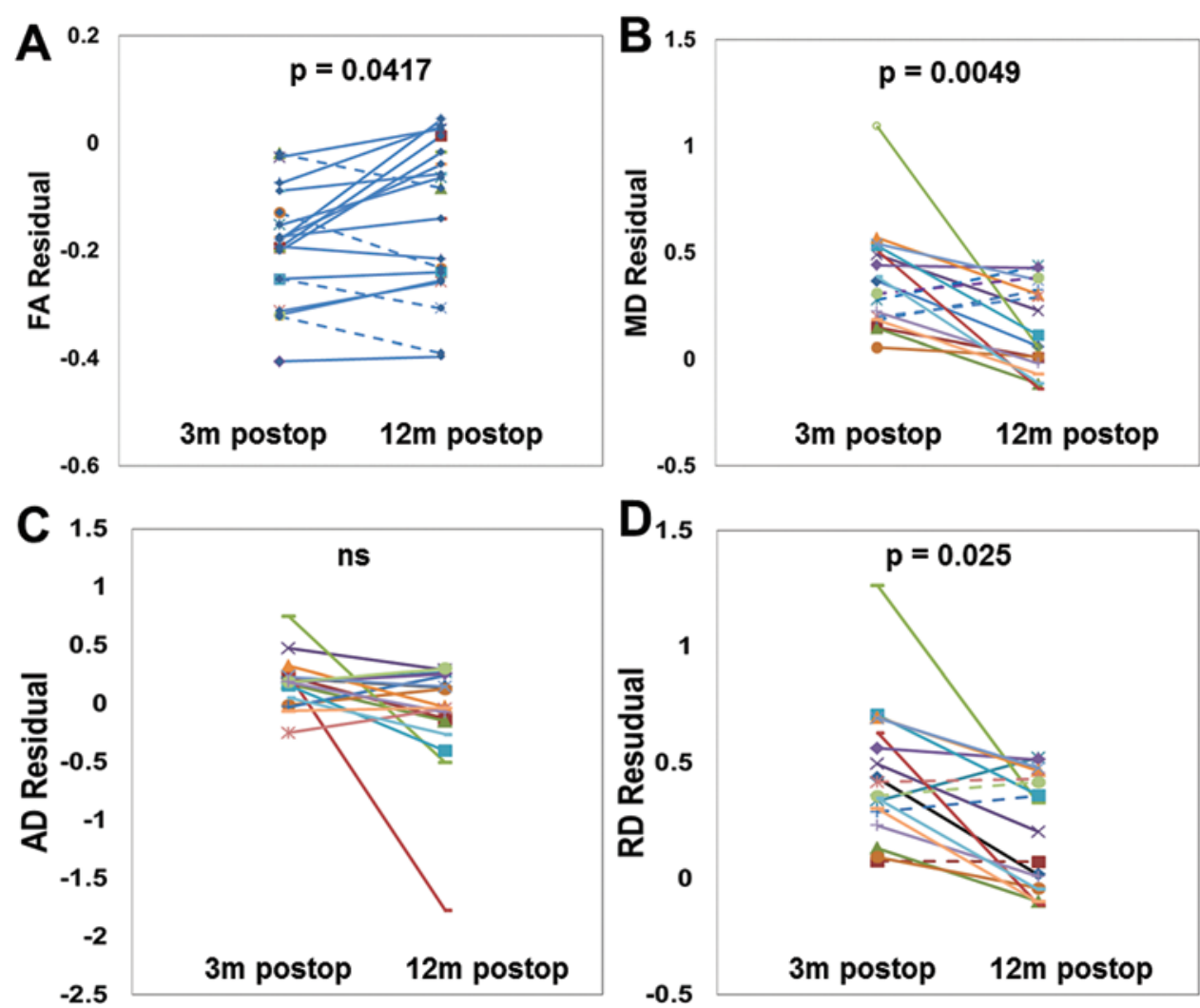

FIG. 5. Longitudinal comparison of DTI residual in the gCC between 3-and 12-month postoperative assessments. A: FA. B: MD. C: AD. D: RD.
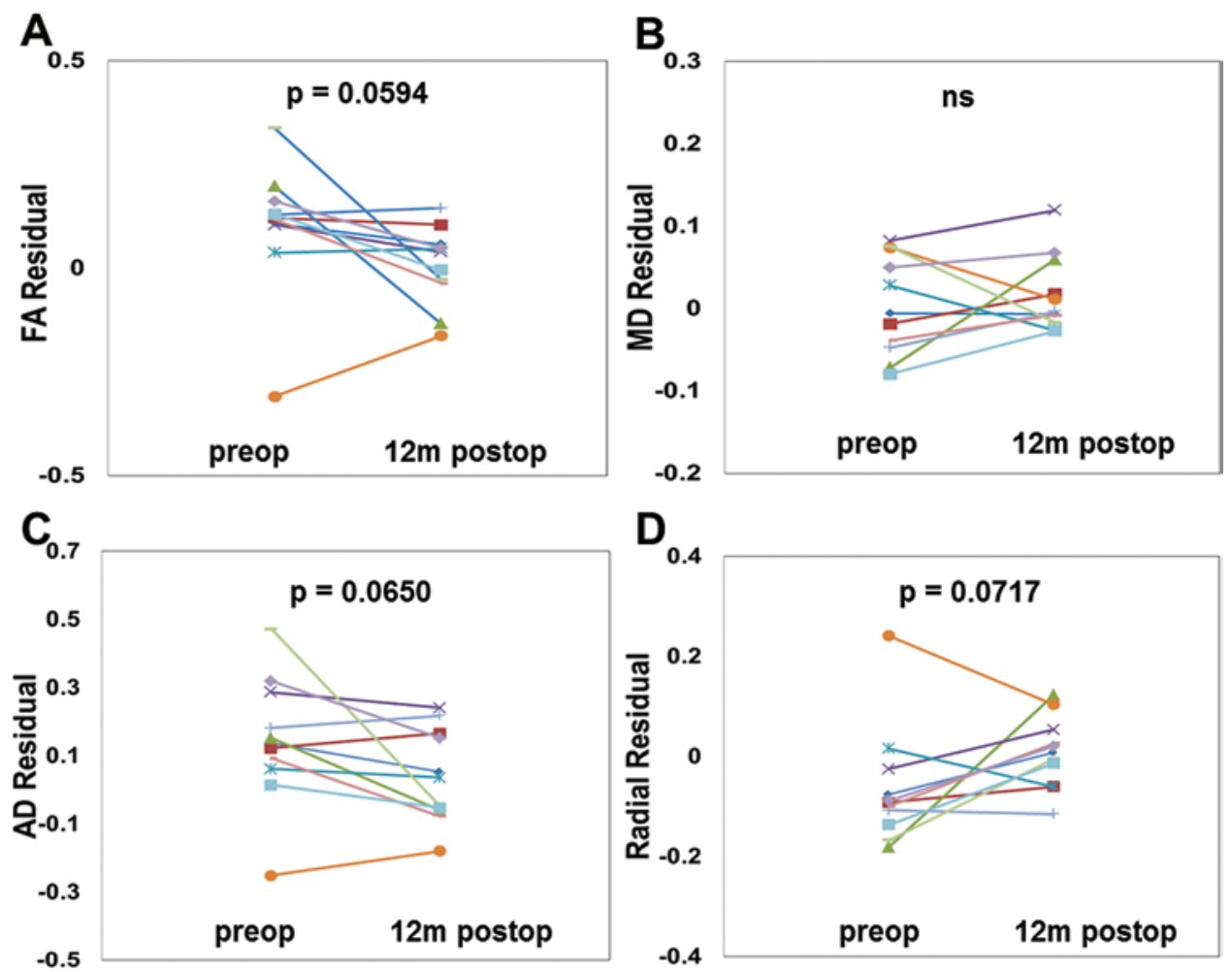

FIG. 6. Longitudinal comparison of DTI residual in the left PLIC between preoperative and 12-month postoperative assessments. A: FA. B: MD. C: AD. D: RD. 
TABLE 5. Comparison of neuropsychological testing results obtained at 3 different time points in patients with hydrocephalus and results in controls*

\begin{tabular}{ccccc}
\hline & & \multicolumn{3}{c}{ Patients w/ HCP } \\
\cline { 3 - 5 } Measure & Controls & Preop & 3 Mos Postop & 12 Mos Postop \\
\hline ABAS-II & & & & \\
\hline GAC & $101.6 \pm 14.2$ & $89.1 \pm 16.9(p=0.0027)$ & $94.9 \pm 15.9(p=0.0562)$ & $85.1 \pm 18.5(p=0.0009)$ \\
\hline Conceptual & $104.6 \pm 13.8$ & $93.5 \pm 17.3(p=0.0065)$ & $99.3 \pm 15.9(p=0.0981)$ & $92.4 \pm 17.7(p=0.0012)$ \\
\hline Social & $100.5 \pm 15.5$ & $91.3 \pm 14.4(p=0.0179)$ & $96.2 \pm 15.0(p=0.0954)$ & $90.3 \pm 17.5(p=0.0097)$ \\
\hline Practical & $99.5 \pm 14.4$ & $91.9 \pm 15.2(p=0.0384)$ & $97.2 \pm 15.6(N S)$ & $84.5 \pm 14.2(p=0.0005)$ \\
\hline Motor Scale & $10.1 \pm 2.9$ & $7.9 \pm 3.9(p=0.0085)$ & $7.9 \pm 3.1(p=0.0020)$ & $6.4 \pm 3.7(p=0.0003)$ \\
\hline Bayley-III & & & & \\
\hline Social & $103.6 \pm 14.9$ & $92.9 \pm 21.0(N S)$ & $95.9 \pm 15.6(p=0.0826)$ & $93.5 \pm 23.8(p=0.0707)$ \\
\hline Cognitive & $99.0 \pm 9.8$ & $89.2 \pm 15.0(p=0.0434)$ & $82.6 \pm 17.2(p=0.0009)$ & $84.2 \pm 17.5(p=0.0002)$ \\
\hline Language & $98.5 \pm 9.2$ & $74.6 \pm 9.1(p=0.0009)$ & $85.2 \pm 16.6(p=0.0005)$ & $80.3 \pm 16.9(p=0.0002)$ \\
\hline Motor & $97.4 \pm 8.7$ & $72.3 \pm 15.0(p=0.0005)$ & $78.9 \pm 18.1(p=0.0003)$ & $75.1 \pm 18.7(p=0.0002)$ \\
\hline
\end{tabular}

* All $p$ values corrected using FDR method. Means are presented \pm SD.

as preterm infants and patients with stroke, traumatic brain injury, and hypoxic-ischemic encephalopathy. ${ }^{1,4,18,24}$ The findings of the current study add new value to the field, demonstrating the predictive and prognostic value of DTI performed at a time shortly after surgical treatment (i.e., 3 months after surgery) for outcomes measures assessed at a later time (12 months after surgery). Whether this relation remains for longer-term prediction and whether DTI at 12 months after injury is a better predictor for future outcome (i.e., beyond 12 months) are yet to be tested. However, our finding shows potential value of DTI in clinical application in the management of postsurgical hydrocephalus pa-

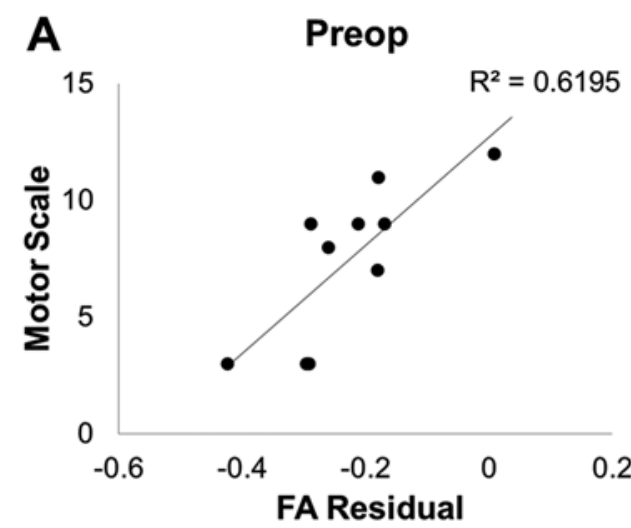

B
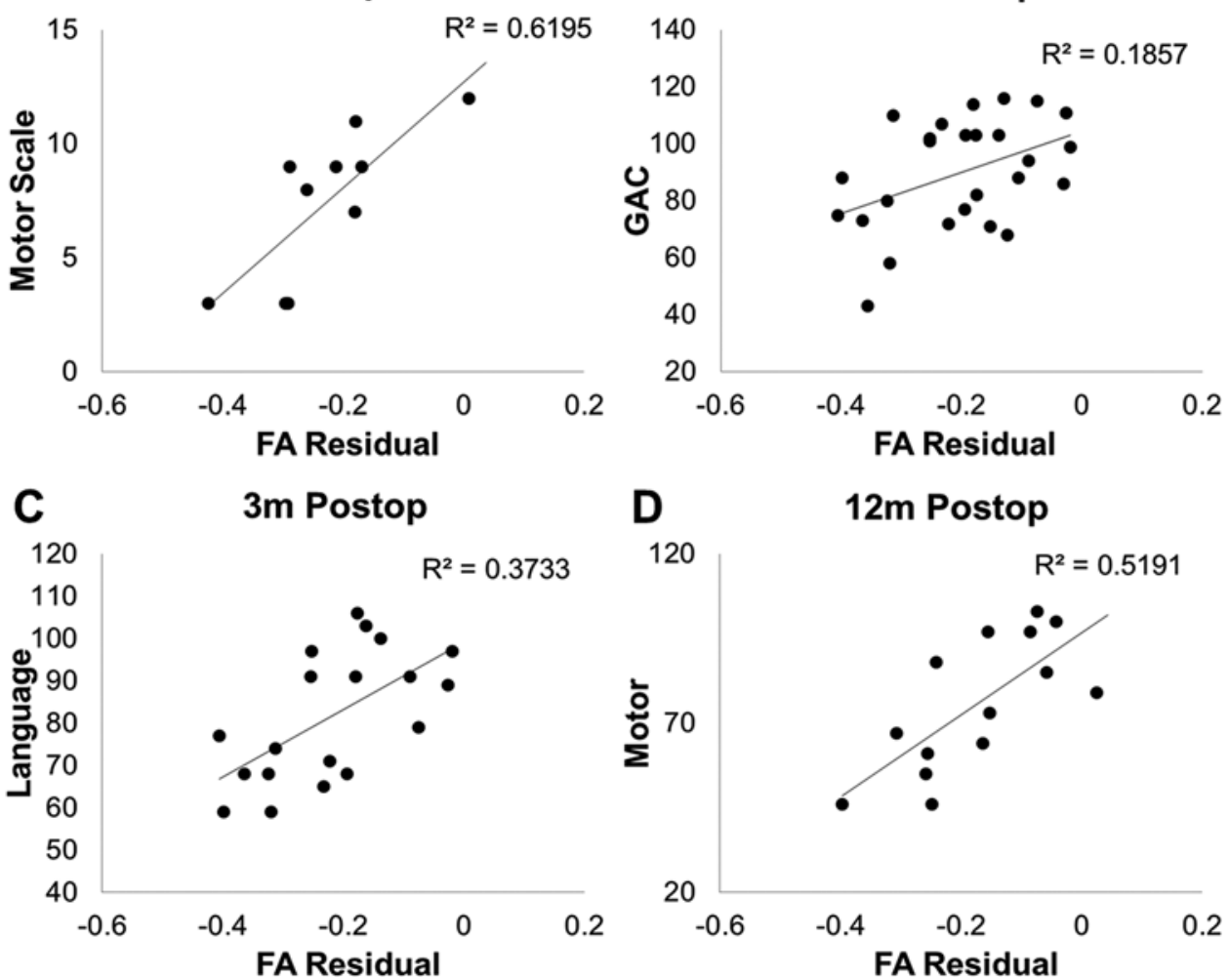

D

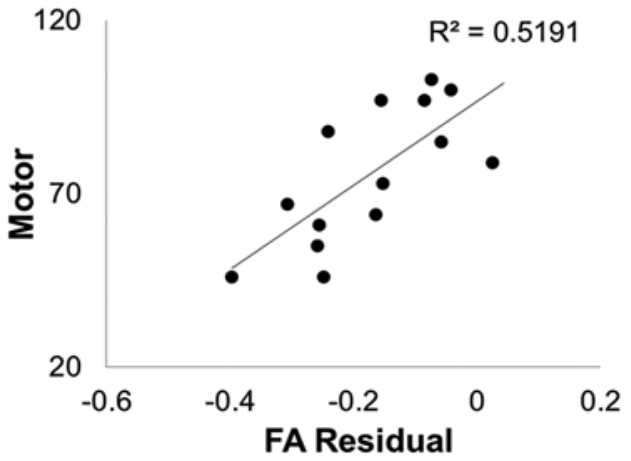

FIG. 7. Correlation of DTI residual in the gCC with neuropsychological outcome assessed at the same time. A: Preoperative assessment: FA residual in the gCC versus ABAS-II Motor Scale. B: Three-month postoperative assessment: FA residual in the gCC versus ABAS-II GAC. C: Three-month postoperative assessment: FA residual in the gCC versus Bayley-III. D: Twelvemonth postoperative assessment: FA residual in the gCC versus Bayley-III Motor. 

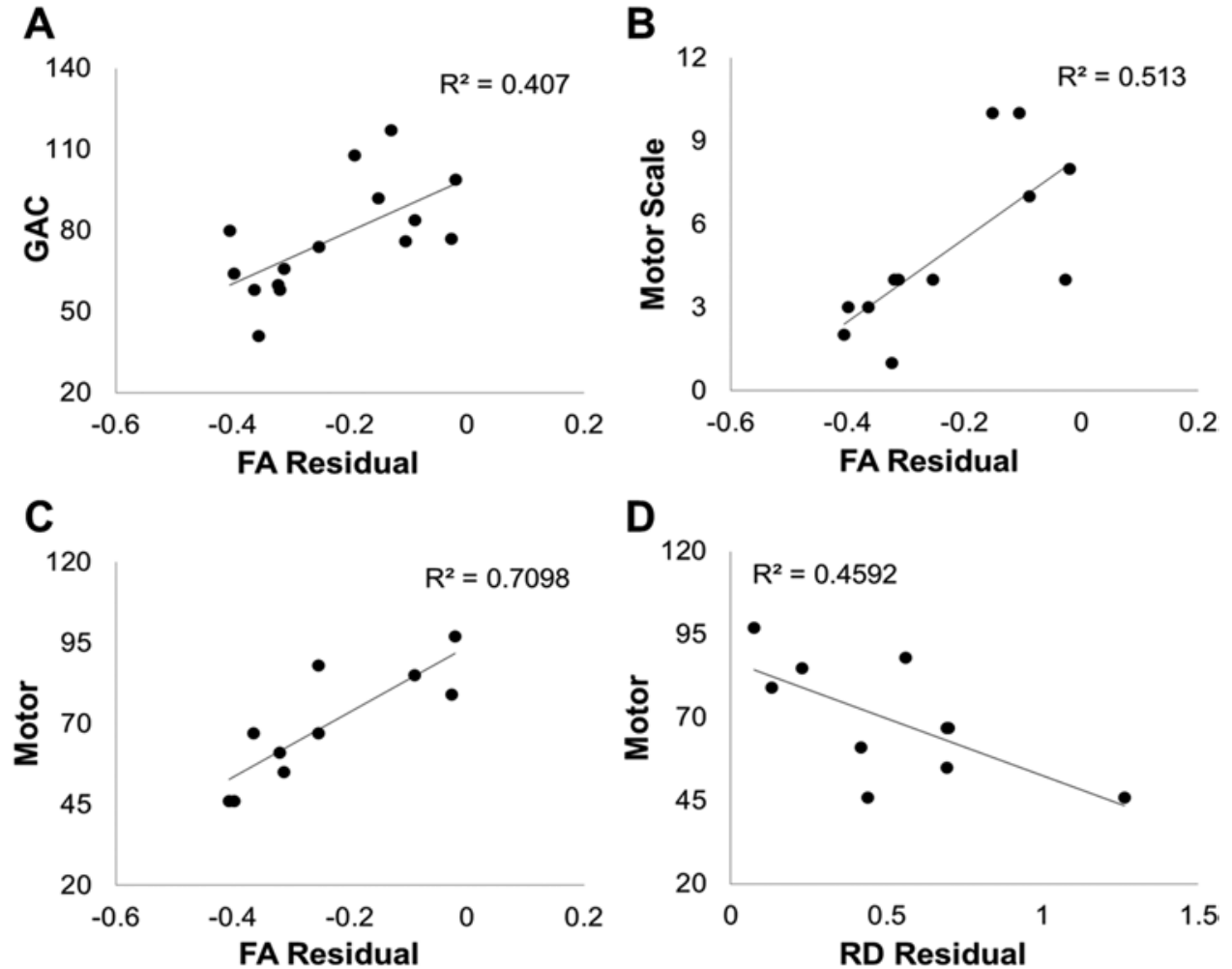

FIG. 8. Correlation of DTI residual in the gCC at 3 months after surgery with neuropsychological outcome at 12 months after surgery. A: FA residual in the gCC versus ABAS-II GAC. B: FA residual in the gCC versus ABAS-II Motor Scale. C: FA residual in the gCC versus Bayley-III Motor. D: RD residual in gCC versus Bayley-III Motor.

tients. Correlations were found between DTI at 3 months after surgery and outcome neuropsychological measures at 12 months after surgery.

DTI may become a valuable tool for clinicians who provide long-term management for patients with hydrocephalus. The study presented here provides initial evidence that would allow the translation of noninvasively obtained radiological information to follow temporal progression of WM integrity in these patients. We generated normative WM maturation curves based on DTI for specific regions, and this can potentially be replicated in other major WM regions or tracts of interest. Creation of such curves will allow us to objectively and noninvasively evaluate patients and eventually extrapolate to future neurocognitive outcomes. When followed longitudinally, the imaging measures will serve to correlate functional outcomes the same way we currently follow head circumference measurements in infants and toddlers to predict normal development or alert us of potential impending clinical pathology.

\section{Limitations}

This study was designed as a prospective longitudinal clinical neuroimaging research project. Due to the logistical difficulty, it was often impractical to enroll and scan patients in a timely manner. Many patients who initially enrolled in the study were not tested (MRI and neuropsychological testing) at all 3 time points, which reduced the power in statistical analysis. The study was designed to include follow-up evaluations at 3 and 12 months after surgery. We found significant DTI abnormalities before surgery and 3 months after surgery in both the gCC and the left PLIC, and the abnormalities persisted in the $\mathrm{gCC}$ at 12 months after surgery. However, we were not able to extrapolate findings from the present study for the trajectory of further recovery after surgery. It would be interesting to follow these surgical patients for a longer period of time to determine when or if the abnormalities (DTI and neuropsychological outcomes) observed in short-term postoperative follow-up will eventually normalize for all patients, and how the correlation between DTI findings at 3 months after surgery and behavioral outcome assessments at 12 months after surgery will evolve temporally in these young patients. A third limitation is the lack of a more comprehensive test battery due to the age range of this patient population. In a recent study, it was found that the nonverbal performance in a cohort of school-age children (6-16 years) with surgically treated hydrocephalus responded well to a short-term occupational therapy intervention targeting the visual spatial, visual attention, and visual motor deficits, which are typical domains of long-term deficits in this patient population. How we can merge the knowledge obtained from the present study, in which most patients were enrolled at $0-3$ years of age, and the other reports of long-term outcome in older children remains a challenge and warrants further investigation. Longer-term follow-up of this cohort would allow addi- 
tional, quantitative neuropsychological scales to be tested to measure performance relative to the performance of healthy peers.

\section{Conclusions}

This is the first prospective neuroimaging study to have systemically investigated WM abnormalities based on DTI in children with hydrocephalus before and after surgery. We found 1) significant WM abnormalities at the acute stage prior to surgery; 2) longitudinal recovery with a trend toward the normal range after surgery; 3 ) persistent WM abnormalities in ROIs tested at 3 months and/or 12 months after surgery; and 4) significant correlation between DTI and developmental outcome measures. These findings provide strong evidence that DTI is a sensitive imaging biomarker for WM abnormalities in pediatric patients with hydrocephalus and potentially can serve as a clinical tool for diagnosis and prognosis in the management of this patient population.

\section{Acknowledgments}

This work was supported by the National Institute of Neurological Disorders and Stroke at the National Institutes of Health (R01 NS066932. PIs: W.Y. and F.T.M.).

\section{References}

1. Aeby A, De Tiège $X$, Creuzil M, David P, Balériaux D, Van Overmeire B, et al: Language development at 2 years is correlated to brain microstructure in the left superior temporal gyrus at term equivalent age: a diffusion tensor imaging study. Neuroimage 78:145-151, 2013

2. Air EL, Yuan W, Holland SK, Jones BV, Bierbrauer K, Altaye $\mathrm{M}$, et al: Longitudinal comparison of pre- and postoperative diffusion tensor imaging parameters in young children with hydrocephalus. J Neurosurg Pediatr 5:385-391, 2010

3. Akbari SH, Limbrick DD Jr, McKinstry RC, Altaye M, Ragan DK, Yuan W, et al: Periventricular hyperintensity in children with hydrocephalus. Pediatr Radiol 45:1189-1197, 2015

4. Ancora G, Testa C, Grandi S, Tonon C, Sbravati F, Savini S, et al: Prognostic value of brain proton MR spectroscopy and diffusion tensor imaging in newborns with hypoxic-ischemic encephalopathy treated by brain cooling. Neuroradiology 55:1017-1025, 2013

5. Aoyama Y, Kinoshita Y, Yokota A, Hamada T: Neuronal damage in hydrocephalus and its restoration by shunt insertion in experimental hydrocephalus: a study involving the neurofilament-immunostaining method. J Neurosurg 104 (5 Suppl):332-339, 2006

6. Assaf Y, Ben-Sira L, Constantini S, Chang LC, Beni-Adani L: Diffusion tensor imaging in hydrocephalus: initial experience. AJNR Am J Neuroradiol 27:1717-1724, 2006

7. Basser PJ, Pierpaoli C: A simplified method to measure the diffusion tensor from seven MR images. Magn Reson Med 39:928-934, 1998

8. Bayley N: The Bayley Scales of Infant and Toddler Development, ed 3. San Antonio, TX: The Psychological Corporation, 2006

9. Beaulieu C: The basis of anisotropic water diffusion in the nervous system - a technical review. NMR Biomed 15:435455, 2002

10. Ben-Sira L, Goder N, Bassan H, Lifshits S, Assaf Y, Constantini S: Clinical benefits of diffusion tensor imaging in hydrocephalus. J Neurosurg Pediatr 16:195-202, 2015

11. Buckley RT, Yuan W, Mangano FT, Phillips JM, Powell S,
McKinstry RC, et al: Longitudinal comparison of diffusion tensor imaging parameters and neuropsychological measures following endoscopic third ventriculostomy for hydrocephalus. J Neurosurg Pediatr 9:630-635, 2012

12. Del Bigio MR, Bruni JE: Periventricular pathology in hydrocephalic rabbits before and after shunting. Acta Neuropathol 77:186-195, 1988

13. Del Bigio MR, Wilson MJ, Enno T: Chronic hydrocephalus in rats and humans: white matter loss and behavior changes. Ann Neurol 53:337-346, 2003

14. Del Bigio MR, Zhang YW: Cell death, axonal damage, and cell birth in the immature rat brain following induction of hydrocephalus. Exp Neurol 154:157-169, 1998

15. Eskandari R, Abdullah O, Mason C, Lloyd KE, Oeschle AN, McAllister JP II: Differential vulnerability of white matter structures to experimental infantile hydrocephalus detected by diffusion tensor imaging. Childs Nerv Syst 30:1651-1661, 2014

16. Eskandari R, McAllister JP II, Miller JM, Ding Y, Ham SD, Shearer DM, et al: Effects of hydrocephalus and ventriculoperitoneal shunt therapy on afferent and efferent connections in the feline sensorimotor cortex. J Neurosurg 101 (2 Suppl):196-210, 2004

17. Fletcher JM, McCauley SR, Brandt ME, Bohan TP, Kramer LA, Francis DJ, et al: Regional brain tissue composition in children with hydrocephalus. Relationships with cognitive development. Arch Neurol 53:549-557, 1996

18. Galanaud D, Perlbarg V, Gupta R, Stevens RD, Sanchez P, Tollard E, et al: Assessment of white matter injury and outcome in severe brain trauma: a prospective multicenter cohort. Anesthesiology 117:1300-1310, 2012

19. Hale PM, McAllister JP II, Katz SD, Wright LC, Lovely TJ, Miller DW, et al: Improvement of cortical morphology in infantile hydrocephalic animals after ventriculoperitoneal shunt placement. Neurosurgery 31:1085-1096, 1992

20. Hannay HJ: Functioning of the corpus callosum in children with early hydrocephalus. J Int Neuropsychol Soc 6:351361, 2000

21. Harrison PL, Oakland T: Adaptive Behavior Assessment System, ed 2. San Antonio, TX: The Psychological Corporation, 2003

22. Hasan KM, Eluvathingal TJ, Kramer LA, Ewing-Cobbs L, Dennis M, Fletcher JM: White matter microstructural abnormalities in children with spina bifida myelomeningocele and hydrocephalus: a diffusion tensor tractography study of the association pathways. J Magn Reson Imaging 27:700-709, 2008

23. Hermoye L, Saint-Martin C, Cosnard G, Lee SK, Kim J, Nassogne MC, et al: Pediatric diffusion tensor imaging: normal database and observation of the white matter maturation in early childhood. Neuroimage 29:493-504, 2006

24. Hirai KK, Groisser BN, Copen WA, Singhal AB, Schaechter JD: Comparing prognostic strength of acute corticospinal tract injury measured by a new diffusion tensor imaging based template approach versus common approaches. J Neurosci Methods 257:204-213, 2016

25. Hirsch JF: Surgery of hydrocephalus: past, present and future. Acta Neurochir (Wien) 116:155-160, 1992

26. Jang SH, Choi BY, Chang CH, Jung YJ, Byun WM, Kim SH, et al: The effects of hydrocephalus on the periventricular white matter in intracerebral hemorrhage: a diffuser tensor imaging study. Int J Neurosci 123:420-424, 2013

27. Jang SH, Ho Kim S: Diffusion tensor imaging following shunt in a patient with hydrocephalus. J Neuroimaging 21:69-72, 2011

28. Jiang H, van Zijl PC, Kim J, Pearlson GD, Mori S: DtiStudio: resource program for diffusion tensor computation and fiber bundle tracking. Comput Methods Programs Biomed 81:106-116, 2006 
29. Khan OH, Enno TL, Del Bigio MR: Brain damage in neonatal rats following kaolin induction of hydrocephalus. Exp Neurol 200:311-320, 2006

30. Kulkarni AV, Donnelly R, Mabbott DJ, Widjaja E: Relationship between ventricular size, white matter injury, and neurocognition in children with stable, treated hydrocephalus. J Neurosurg Pediatr 16:267-274, 2015

31. Lovely TJ, McAllister JP II, Miller DW, Lamperti AA, Wolfson BJ: Effects of hydrocephalus and surgical decompression on cortical norepinephrine levels in neonatal cats. Neurosurgery 24:43-52, 1989

32. Mataró M, Junqué C, Poca MA, Sahuquillo J: Neuropsychological findings in congenital and acquired childhood hydrocephalus. Neuropsychol Rev 11:169-178, 2001

33. Olopade FE, Shokunbi MT, Sirén AL: The relationship between ventricular dilatation, neuropathological and neurobehavioural changes in hydrocephalic rats. Fluids Barriers CNS 9:19, 2012

34. Rajagopal A, Shimony JS, McKinstry RC, Altaye M, Maloney T, Mangano FT, et al: White matter microstructural abnormality in children with hydrocephalus detected by probabilistic diffusion tractography. AJNR Am J Neuroradiol 34:2379-2385, 2013

35. Röricht S, Meyer BU, Woiciechowsky C, Lehmann R: Callosal and corticospinal tract function in patients with hydrocephalus: a morphometric and transcranial magnetic stimulation study. J Neurol 245:280-288, 1998

36. Scheel M, Diekhoff T, Sprung C, Hoffmann KT: Diffusion tensor imaging in hydrocephalus - findings before and after shunt surgery. Acta Neurochir (Wien) 154:1699-1706, 2012

37. Sun M, Yuan W, Hertzler DA, Cancelliere A, Altaye M, Mangano FT: Diffusion tensor imaging findings in young children with benign external hydrocephalus differ from the normal population. Childs Nerv Syst 28:199-208, 2012

38. Ware AL, Juranek J, Williams VJ, Cirino PT, Dennis M, Fletcher JM: Anatomical and diffusion MRI of deep gray matter in pediatric spina bifida. Neuroimage Clin 5:120127,2014

39. Woods RP, Grafton ST, Watson JD, Sicotte NL, Mazziotta JC: Automated image registration: II. Intersubject validation of linear and nonlinear models. J Comput Assist Tomogr 22:153-165, 1998

40. Yuan W, Mangano FT, Air EL, Holland SK, Jones BV, Altaye $\mathrm{M}$, et al: Anisotropic diffusion properties in infants with hydrocephalus: a diffusion tensor imaging study. AJNR Am J Neuroradiol 30:1792-1798, 2009

41. Yuan W, Deren KE, McAllister JP II, Holland SK, Lindquist DM, Cancelliere A, et al: Diffusion tensor imaging correlates with cytopathology in a rat model of neonatal hydrocephalus. Cerebrospinal Fluid Res 7:19, 2010

42. Yuan W, McAllister JP II, Lindquist DM, Gill N, Holland
SK, Henkel D, et al: Diffusion tensor imaging of white matter injury in a rat model of infantile hydrocephalus. Childs Nerv Syst 28:47-54, 2012

43. Yuan W, McAllister JP II, Mangano FT: Neuroimaging of white matter abnormality in pediatric hydrocephalus. J Pediatr Neuroradiol 2:119-128, 2013

44. Yuan W, McKinstry RC, Shimony JS, Altaye M, Powell SK, Phillips JM, et al: Diffusion tensor imaging properties and neurobehavioral outcomes in children with hydrocephalus. AJNR Am J Neuroradiol 34:439-445, 2013

45. O’Hayon BB, Drake JM, Ossip MG, Tuli S, Clarke M: Frontal and occipital horn ratio: A linear estimate of ventricular size for multiple imaging modalities in pediatric hydrocephalus. Pediatr Neurosurg 29:245-249, 1998

\section{Disclosures}

Dr. McKinstry reports receiving acting/modeling fees, honoraria, and travel support from Siemens, unrelated to this manuscript.

\section{Author Contributions}

Conception and design: Yuan, Mangano, McKinstry, Holland. Acquisition of data: Powell, Phillips, Barnard, Dodd, Simpson, Mercer, Bidwell. Analysis and interpretation of data: Yuan, Mangano, Altaye, McKinstry, Shimony, Powell, Phillips, Barnard, Limbrick, Holland, Jones, Dodd. Drafting the article: Yuan, Mangano. Critically revising the article: Yuan, Mangano, Altaye, Shimony, Limbrick, Holland, Jones, Dodd. Reviewed submitted version of manuscript: all authors. Approved the final version of the manuscript on behalf of all authors: Yuan. Statistical analysis: Yuan, Altaye. Administrative/technical/material support: Simpson, Mercer, Rajagopal, Bidwell. Study supervision: Yuan, Mangano, McKinstry, Powell, Limbrick, Jones. Data processing: Simpson, Rajagopal

\section{Supplemental Information \\ Online-Only Content}

Supplemental material is available with the online version of the article.

Supplemental Table 1. http://thejns.org/doi/suppl/10.3171/ 2016.2.PEDS15628.

\section{Correspondence}

Weihong Yuan, Division of Radiology, Pediatric Neuroimaging Research Consortium, Cincinnati Children's Hospital Medical Center, 3333 Burnet Ave., MLC 5033, Cincinnati, OH 45229. email: weihong.yuan@cchmc.org. 